\title{
Sd-model with strong exchange coupling and a metal-insulator phase transition
}

\author{
Yu.A.Izyumov, N.I.Chaschin, D.S.Alexeev \\ Institute for Metal Physics, S.Kovalevskaya str., 18, Ekaterinburg, Russia
}

Received May 18, 2006, in final form June 14, 2006

\begin{abstract}
Sd-exchange model (Kondo lattice model) is formulated for strong sd-coupling within the framework of the $X$ operators technique and the generating functional approach. The $X$-operators are constructed based on the exact eigen functions of a single-site sd-exchange Hamiltonian. Such representation enables us to develop a perturbation theory near the atomic level. A locator-type representation was derived for the electron Green's function. The electron self-energy includes interaction of electrons and spin fluctuations. An integral equation for the self-energy was obtained in the limit of infinite localized spins. A solution of this equation in the static approximation for spin fluctuations leads to a structure of electron Green's function showing a metal-insulator phase transition. This transition is similar to that in the Hubbard model at half filling.
\end{abstract}

Key words: Kondo lattice model, strongly correlated electron systems, metal-insulator phase transitions

PACS: $71.10 .-w, 71.10 . F d, 71.27 .+a$

\section{Introduction}

The sd-exchange model is one of the fundamental models in the theory of strongly correlated systems. This model is frequently referred to as the Kondo lattice model in the West. Its Hamiltonian takes into account the hopping of conduction electrons over a lattice and the exchange interaction of them with the localized atomic moments (value spin $S$ ) placed at the lattice sites. The Hamiltonian is written as

$$
\mathcal{H}=\sum_{i j \sigma} t_{i j} c_{i \sigma}^{\dagger} c_{i \sigma}-\frac{J}{2} \sum_{i}\left(\boldsymbol{S}_{i} \boldsymbol{\sigma}_{i}\right) .
$$

Here $c_{i \sigma}\left(c_{i \sigma}^{\dagger}\right)$ is a Fermi-operator of annihilation (creation) of an electron on a site $i$ with spin projection $\sigma$.

For the case of strong sd-exchange coupling $S|J| \gg W$ (W is width of the electron band) the sd-model was applied for description of a metal-insulator phase transition [1,2] and physics of magnetic semiconductors [3]. Exact solution of eigenvalue problem for a single site exchange Hamiltonian was applied [1-3]. Two energy levels $E_{+}$and $E_{-}$for the states with the total spin on a site $j_{+}=S+1 / 2$ and $j_{+}=S-1 / 2$ are spread into two bands by hopping term, that determines the physics of the model in the strong coupling limit.

In recent works $[4,5]$ we applied the generating functional approach (GFA) to this problem which, in a most general form, permits to construct a perturbation theory "near the atomic limit". Equations for electron Green's functions (GF) were derived in terms of functional derivatives over fluctuating fields introduced in GFA, and the simplest approximations for their solution were tested. In the present paper we return to the problem on a most general basis. Particularly, we introduced four-component electron operator instead of two-component one used in works $[4,5]$. Such generalization of mathematical structure makes it possible to construct more correctly the perturbation theory near the atomic limit and to consider the states with more complicated order parameters such as magnetic ordering or superconductivity. 
In the case of strong sd-exchange coupling one has to use the exact solution of the problem on one site. Wave functions of the Hamiltonian $J / 2(\boldsymbol{S} \boldsymbol{\sigma})$ are known [1,2]:

$$
\begin{aligned}
& |\mathrm{M} 0\rangle=|\mathrm{M}\rangle|0\rangle, \\
& |\mathrm{M} 2\rangle=|\mathrm{M}\rangle|2\rangle \\
& \left|\mathrm{M}^{\alpha} \alpha\right\rangle=\sum_{\sigma} \Theta^{\sigma \alpha}\left(\mathrm{M}^{\alpha}\right)\left|\mathrm{M}^{\alpha}-\frac{\sigma}{2}\right\rangle c_{\sigma}^{\dagger}|0\rangle, \quad \alpha=+,-,
\end{aligned}
$$

where

$$
\Theta^{\sigma+}=\sqrt{\frac{S+\sigma \mathrm{M}+\frac{1}{2}}{2 S+1}}, \quad \Theta^{\sigma-}=\sigma \sqrt{\frac{S+\bar{\sigma} \mathrm{M}+\frac{1}{2}}{2 S+1}} .
$$

A wave function $|\mathrm{M} 0\rangle$ describes the state of a site without an electron when the atom is in $|\mathrm{M}\rangle$ state with projection of spin $\mathrm{M} ;|\mathrm{M} 2\rangle$ is a state with two electrons on a site, and $\left|\mathrm{M}^{\alpha} \alpha\right\rangle$ with only one electron with projection of total spin $j_{\alpha}$ and

$$
-\left(S+\frac{\alpha}{2}\right)<\cdots<\mathrm{M}^{\alpha}<\cdots<\left(S+\frac{\alpha}{2}\right), \quad j_{\alpha}=S+\frac{\alpha}{2} .
$$

In $\left|\mathrm{M}^{\alpha} \alpha\right\rangle$ state the wave function is a superposition with both projections of an electron spin with mixing coefficients being Klebs-Gordan coefficients. Selfenergy of one site exchange Hamiltonian is as follows:

$$
\left.\begin{array}{ll}
E^{+}=-\frac{1}{2} J S, & j=S+\frac{1}{2} \\
E^{-}=\frac{1}{2} J(S+1), & j=S-\frac{1}{2}
\end{array}\right\} .
$$

Because the self-energy is known it is possible to develop a perturbation theory over small parameter $W / J S$. The best way to do it is GFA combined with the formalism of $X$-operators.

\section{Formalism of $X$-operators}

Any one-site operator $\widehat{A}_{i}$ can be expanded over the system of $X$-operators

$$
X_{i}^{p q}=|p\rangle\langle q|
$$

determined on the base of functions $|p\rangle$. This explanation is written as

$$
\widehat{A}_{i}=\sum_{p q}\langle p|\widehat{A}| q\rangle X_{i}^{p q} .
$$

The calculation of a matrix element of an annihilation operator $c_{i \sigma}$ leads to its representation in terms of $X$-operators $[2,4-7]$ :

$$
c_{i \sigma}=\sum_{\mathrm{M} \alpha}\left[\Theta^{\sigma \alpha}\left(\mathrm{M}+\frac{\sigma}{2}\right) X_{i}^{\mathrm{M} 0 ;\left(\mathrm{M}+\frac{\sigma}{2}\right) \alpha}+\sigma \Theta^{\bar{\sigma} \alpha}\left(\mathrm{M}-\frac{\sigma}{2}\right) X_{i}^{\left(\mathrm{M}-\frac{\sigma}{2}\right) \alpha ; \mathrm{M} 2}\right] .
$$

Let us introduce four-component construction of Fermi-like $X$-operators

$$
\Psi_{1 \sigma}(\mathrm{I})=\left(\begin{array}{c}
X_{1}^{\mathrm{M} 0} ;\left(\mathrm{M}+\frac{\sigma}{2}\right)+ \\
\sigma X_{1}^{\left(\mathrm{M}-\frac{\sigma}{2}\right)+; \mathrm{M} 2} \\
X_{1}^{\mathrm{M} 0} ;\left(\mathrm{M}+\frac{\sigma}{2}\right)- \\
\sigma X_{1}^{\left(\mathrm{M}-\frac{\sigma}{2}\right)-; \mathrm{M} 2}
\end{array}\right),
$$

as well as a line $\Psi_{i \sigma}^{\dagger}(\mathrm{I})$ including the conjugated $X$-operators. Operator $\Psi_{1 \sigma}(\mathrm{I})$ describes the creation of an electron on site $i$ at time $\tau(1=\mathrm{i} \tau)$ with spin $\sigma$ when the atom has an angular momentum 
projection $\mathrm{M}$, with two possible combinations of these momenta labeled by indexes $\alpha=+,-$. Components of spinor are numbered by index $\nu=1,2,3,4$. Its combined index $\mathrm{I}=(\mathrm{M} \nu)$.

In such representation the Hamiltonian of sd-model is written as

$$
\begin{aligned}
\mathcal{H} & =\mathcal{H}_{s d}-\mu N=\mathcal{H}_{1}+\mathcal{H}_{2} \\
\mathcal{H}_{1} & =-2 \sum_{i \mathrm{M}} \mu X_{i}^{\mathrm{M} 2 ; \mathrm{M} 2}+\sum_{i \alpha \mathrm{M}^{\alpha}}\left(E^{\alpha}-\mu\right) X_{i}^{\mathrm{M}^{\alpha} \alpha ; \mathrm{M}^{\alpha} \alpha}, \\
\mathcal{H}_{2} & =\sum_{i j} t_{i j} \sum_{\sigma_{1} \mathrm{I}_{1} \sigma_{2} \mathrm{I}_{2}} \Psi_{1 \sigma_{1}}^{\dagger}\left(\mathrm{I}_{1}\right) \mathbb{T}\left(\sigma_{1} \mathrm{I}_{1} ; \sigma_{2} \mathrm{I}_{2}\right) \Psi_{j \sigma_{2}}\left(\mathrm{I}_{2}\right) .
\end{aligned}
$$

where

$$
\begin{aligned}
& \mathbb{T}\left(\sigma_{1} \mathrm{I}_{1} ; \sigma_{2} \mathrm{I}_{2}\right)=\delta_{\sigma_{1} \sigma_{2}} \vartheta^{\sigma_{1}}\left(\mathrm{I}_{1}\right) \mathrm{T} \vartheta^{\sigma_{2}}\left(\mathrm{I}_{2}\right), \\
& \vartheta^{\sigma}(\mathrm{I})=\operatorname{diag}\left\{\Theta^{\sigma+}\left(\mathrm{M}+\frac{\sigma}{2}\right), \Theta^{\bar{\sigma}+}\left(\mathrm{M}-\frac{\sigma}{2}\right), \Theta^{\sigma-}\left(\mathrm{M}+\frac{\sigma}{2}\right), \Theta^{\bar{\sigma}-}\left(\mathrm{M}-\frac{\sigma}{2}\right)\right\},
\end{aligned}
$$

and $\mathrm{T}$ is a $4 \times 4$ matrix with all elements being equal to 1 .

Let us introduce a supermatrix GF

$$
\mathcal{L}_{12}^{\sigma_{1} \sigma_{2}}\left(\mathrm{I}_{1}, \mathrm{I}_{2}\right)=-\left(\begin{array}{ll}
\left\langle T \Psi_{1 \sigma_{1}}\left(\mathrm{I}_{1}\right) \Psi_{2 \sigma_{2}}^{\dagger}\left(\mathrm{I}_{2}\right)\right\rangle_{V} & \left\langle T \Psi_{1 \sigma_{1}}\left(\mathrm{I}_{1}\right) \Psi_{2 \sigma_{2}}\left(\mathrm{I}_{2}\right)\right\rangle_{V} \\
\left\langle T \Psi_{1 \sigma_{1}}^{\dagger}\left(\mathrm{I}_{1}\right) \Psi_{2 \sigma_{2}}^{\dagger}\left(\mathrm{I}_{2}\right)\right\rangle_{V} & \left\langle T \Psi_{1 \sigma_{1}}^{\dagger}\left(\mathrm{I}_{1}\right) \Psi_{2 \sigma_{2}}\left(\mathrm{I}_{2}\right)\right\rangle_{V}
\end{array}\right)
$$

each of its elements is a matrix $4 \times 4$.

Here $\langle T \cdots\rangle_{V}$ is a statistical average of some chronological product of operators of the system in fluctuating fields depending on both sites and times

$$
\langle T \cdots\rangle_{V}=\frac{1}{Z[V]} \operatorname{Tr}\left(\mathrm{e}^{-\beta \mathcal{H}} T \cdots \mathrm{e}^{-V}\right)
$$

where

$$
Z[V]=\operatorname{Tr}\left(\mathrm{e}^{-\beta \mathcal{H}} T \mathrm{e}^{-V}\right)
$$

is the partition sum which is a functional of the fluctuating fields. $V$ is the operator of interaction of the fields with our system. According to general concept GFA operator $V$ is a linear combination of bose-like $X$-operators and the mixing coefficients are just the fluctuating fields. In our case $V$ is taken in a form:

$$
\begin{aligned}
V= & v_{1^{\prime}}^{\mathrm{M}_{1}^{\prime} 0 ; \mathrm{M}_{1}^{\prime} 0} X_{1^{\prime}}^{\mathrm{M}_{1}^{\prime} 0 ; \mathrm{M}_{1}^{\prime} 0}+v_{1^{\prime}}^{\mathrm{M}_{1}^{\prime} 2 ; \mathrm{M}_{1}^{\prime} 2} X_{1^{\prime}}^{\mathrm{M}_{1}^{\prime} 2 ; \mathrm{M}_{1}^{\prime} 2}+v_{1^{\prime}}^{\mathrm{M}_{1}^{\prime} 0 ; \mathrm{M}_{1}^{\prime} 2} X_{1^{\prime}}^{\mathrm{M}_{1}^{\prime} 2 ; \mathrm{M}_{1}^{\prime} 0}+v_{1^{\prime}}^{\mathrm{M}_{1}^{\prime} 2 ; \mathrm{M}_{1}^{\prime} 0} X_{1^{\prime}}^{\mathrm{M}_{1}^{\prime} 0 ; \mathrm{M}_{1}^{\prime} 2} \\
& +v_{1^{\prime}}^{\left(\mathrm{M}_{1}^{\prime}+\frac{\sigma_{2}^{\prime}}{2}\right) \alpha_{2}^{\prime} ;\left(\mathrm{M}_{1}^{\prime}+\frac{\sigma_{1}^{\prime}}{2}\right) \alpha_{1}^{\prime}} X_{1^{\prime}}^{\left(\mathrm{M}_{1}^{\prime}+\frac{\sigma_{1}^{\prime}}{2}\right) \alpha_{1}^{\prime} ;\left(\mathrm{M}_{1}^{\prime}+\frac{\sigma_{2}^{\prime}}{2}\right) \alpha_{2}^{\prime}} .
\end{aligned}
$$

Herein below a sum over the repeated primed indexes is implied. The rest of the definition corresponds to the standard technique of the temperature GFs [8].

The introduction of the fluctuating fields makes it possible to derive an equation of motion for electron GF in terms of functional derivatives with respect to these fields. This is a principle of GFA.

We have to write an equation of motion for each matrix element of GF (2.9). For example for an element " 11 " a general form of the equation is:

$$
\begin{aligned}
\frac{\partial}{\partial \tau_{1}}\left(\left(T \Psi_{1 \sigma_{2}}\left(\mathrm{I}_{1}\right) \Psi_{2 \sigma_{2}}^{\dagger}\left(\mathrm{I}_{2}\right)\right)\right)_{V}= & \delta\left(\tau_{1}-\tau_{2}\right)\left(\left(T\left[\Psi_{1 \sigma_{2}}\left(\mathrm{I}_{1}\right), \Psi_{2 \sigma_{2}}^{\dagger}\left(\mathrm{I}_{2}\right)\right]_{+}\right)\right)_{V} \\
& +\left(\left(T \dot{\Psi}_{1 \sigma_{2}}\left(\mathrm{I}_{1}\right) \Psi_{2 \sigma_{2}}^{\dagger}\left(\mathrm{I}_{2}\right)\right)\right)_{V}-\left(\left(T\left[\Psi_{1 \sigma_{2}}\left(\mathrm{I}_{1}\right), V\right]_{-} \Psi_{2 \sigma_{2}}^{\dagger}\left(\mathrm{I}_{2}\right)\right)\right)_{V},
\end{aligned}
$$

where we introduced a short notation

$$
((T \ldots))_{V}=\operatorname{Tr}\left(\mathrm{e}^{-\beta \mathcal{H}} T \ldots \mathrm{e}^{-V}\right) .
$$


Equation of motion for operator $\Psi_{1 \sigma_{1}}\left(\mathrm{M}_{1}\right)$ can be written in a form:

$$
\dot{\Psi}_{1}\left(\mathrm{I}_{1}\right)=-\mathcal{E}\left(\mathrm{I}_{1} \mathrm{I}_{1}^{\prime}\right) \Psi_{1}\left(\mathrm{I}_{1}^{\prime}\right)-t_{11^{\prime}} \mathcal{F}_{1}\left(\mathrm{I}_{1} \mathrm{I}_{2}^{\prime}\right) \mathbb{T}\left(\mathrm{I}_{2}^{\prime} \mathrm{I}_{1}^{\prime}\right) \Psi_{1^{\prime}}\left(\mathrm{I}_{1}^{\prime}\right)+\mathcal{Q}_{1}\left(\mathrm{I}_{1} \mathrm{I}_{2}^{\prime}\right) \mathbb{T}\left(\mathrm{I}_{2}^{\prime} \mathrm{I}_{1}^{\prime}\right) \Psi_{1^{\prime}}^{\dagger}\left(\mathrm{I}_{1}^{\prime}\right) t_{1^{\prime} 1} .
$$

Here we use a combined index $\mathrm{I}(\sigma \mathrm{M})$ and introduce quantities $\mathcal{F}_{1}$ and $\mathcal{Q}_{1}$ appearing from anticommutators of electron operators $\Psi_{1}\left(\mathrm{I}_{1}\right)$ and $\Psi_{2}^{\dagger}\left(\mathrm{I}_{2}\right)$ (here spin index $\sigma$ is included in I):

$$
\left.\begin{array}{l}
{\left[\Psi_{1}\left(\mathrm{I}_{1}\right), \Psi_{2}^{\dagger}\left(\mathrm{I}_{2}\right)\right]_{+}=\delta_{12} \mathcal{F}_{1}\left(\mathrm{I}_{1} \mathrm{I}_{2}\right)} \\
{\left[\Psi_{1}\left(\mathrm{I}_{1}\right), \Psi_{2}\left(\mathrm{I}_{2}\right)\right]_{+}=\delta_{12} \mathcal{Q}_{1}\left(\mathrm{I}_{1} \mathrm{I}_{2}\right)}
\end{array}\right\}
$$

$\mathcal{F}_{1}$ and $\mathcal{Q}_{1}$ are $4 \times 4$ matrices which we denote as supermatrices

$$
\mathcal{F}=\left(\begin{array}{ll}
\mathcal{F}^{11} & \mathcal{F}^{12} \\
\mathcal{F}^{21} & \mathcal{F}^{22}
\end{array}\right), \quad \mathcal{Q}=\left(\begin{array}{cc}
\mathcal{Q}^{11} & 0 \\
0 & \mathcal{Q}^{11}
\end{array}\right)
$$

with matrix elements:

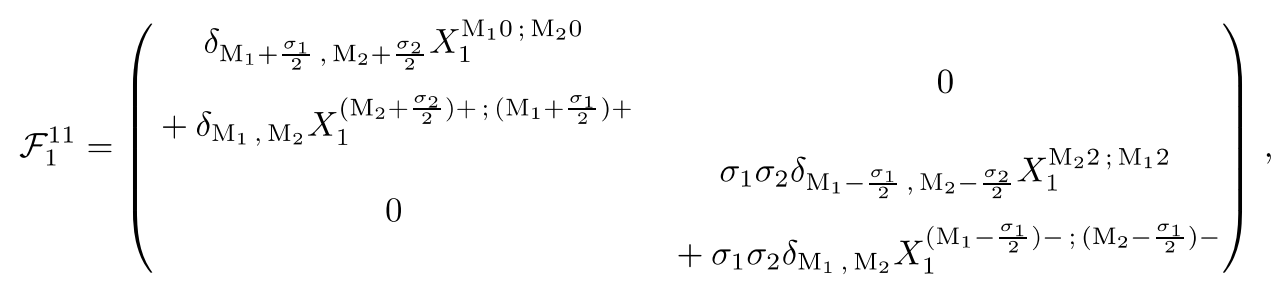

$$
\begin{aligned}
& \mathcal{F}_{1}^{12}=\left(\begin{array}{cc}
\delta_{\mathrm{M}_{1}, \mathrm{M}_{2}} X_{1}^{\left(\mathrm{M}_{1}+\frac{\sigma_{1}}{2}\right)-;\left(\mathrm{M}_{1}+\frac{\sigma_{1}}{2}\right)+} & 0 \\
0 & \sigma_{1} \sigma_{2} \delta_{\mathrm{M}_{1}, \mathrm{M}_{2}} X_{1}^{\left(\mathrm{M}_{1}-\frac{\sigma_{1}}{2}\right)+;\left(\mathrm{M}_{1}-\frac{\sigma_{1}}{2}\right)-}
\end{array}\right) .
\end{aligned}
$$

Matrix elements $\mathcal{F}_{1}^{21}$ and $\mathcal{F}_{1}^{22}$ can be obtained from $\mathcal{F}_{1}^{12}$ and $\mathcal{F}_{1}^{11}$ replacing $\alpha$ by $\bar{\alpha}$ and replacing $X^{\mathrm{M} 0} ; \mathrm{M} 0$ by $X^{\mathrm{M} 2 ; \mathrm{M} 2}$.

Matrix $\mathcal{Q}$ is a block-diagonal one and nonzero matrix block is

$$
\mathcal{Q}_{1}^{11}=\left(\begin{array}{cc}
0 & \sigma_{2} \delta_{\mathrm{M}_{1}+\frac{\sigma_{1}}{2}, \mathrm{M}_{2}-\frac{\sigma_{2}}{2}} X_{1}^{\mathrm{M}_{1} 0 ; \mathrm{M}_{2} 2} \\
\sigma_{1} \delta_{\mathrm{M}_{1}-\frac{\sigma_{1}}{2}, \mathrm{M}_{2}+\frac{\sigma_{2}}{2}} X_{1}^{\mathrm{M}_{2} 0 ; \mathrm{M}_{1} 2} & 0
\end{array}\right) .
$$

As one can see, diagonal elements of matrix $\mathcal{F}$ conserve the states with total momentum $j_{\alpha}$ but off diagonal ones mix them up. Matrix $\mathcal{Q}$ deals with transitions between empty sites and double occupied sites.

Matrices $\mathcal{F}_{1}^{\dagger}\left(\mathrm{I}_{1} \mathrm{I}_{2}\right)$ and $\mathcal{Q}_{1}^{\dagger}\left(\mathrm{I}_{1} \mathrm{I}_{2}\right)$ are obtained from matrices $\mathcal{F}_{1}\left(\mathrm{I}_{1} \mathrm{I}_{2}\right)$ and $\mathcal{Q}_{1}\left(\mathrm{I}_{1} \mathrm{I}_{2}\right)$ by taking hermitian conjugation of $X$-operator and changing indexes $\mathrm{I}_{1} \leftrightarrow \mathrm{I}_{2}$.

Let us now explain the rest of notations in equation (2.15).

$$
\mathcal{E}\left(\mathrm{I}_{1} \mathrm{I}_{2}\right)=\delta_{\sigma_{1} \sigma_{2}} \delta_{\mathrm{M}_{1}, \mathrm{M}_{2}} \operatorname{diag}\left\{E_{+}-\mu,-E_{+}-\mu, E_{-}-\mu,-E_{-}-\mu\right\} \equiv \delta_{\sigma_{1} \sigma_{2}} \delta_{\mathrm{M}_{1}, \mathrm{M}_{2}} \mathcal{E}
$$

And finally, we have to present a commutator of operator $\Psi_{1}$ with fluctuating fields:

$$
\left[\Psi_{1}\left(\mathrm{I}_{1}\right), V\right]=W_{1}\left(\mathrm{I}_{1} \mathrm{I}_{1}^{\prime}\right) \Psi_{1}\left(\mathrm{I}_{1}^{\prime}\right)+\Psi^{\dagger}\left(\mathrm{I}_{1}^{\prime}\right)\left(W_{1}^{02}\right)\left(\mathrm{I}_{1}^{\prime} \mathrm{I}_{1}\right),
$$

where $W$ and $W^{02}$ are $4 \times 4$-matrices:

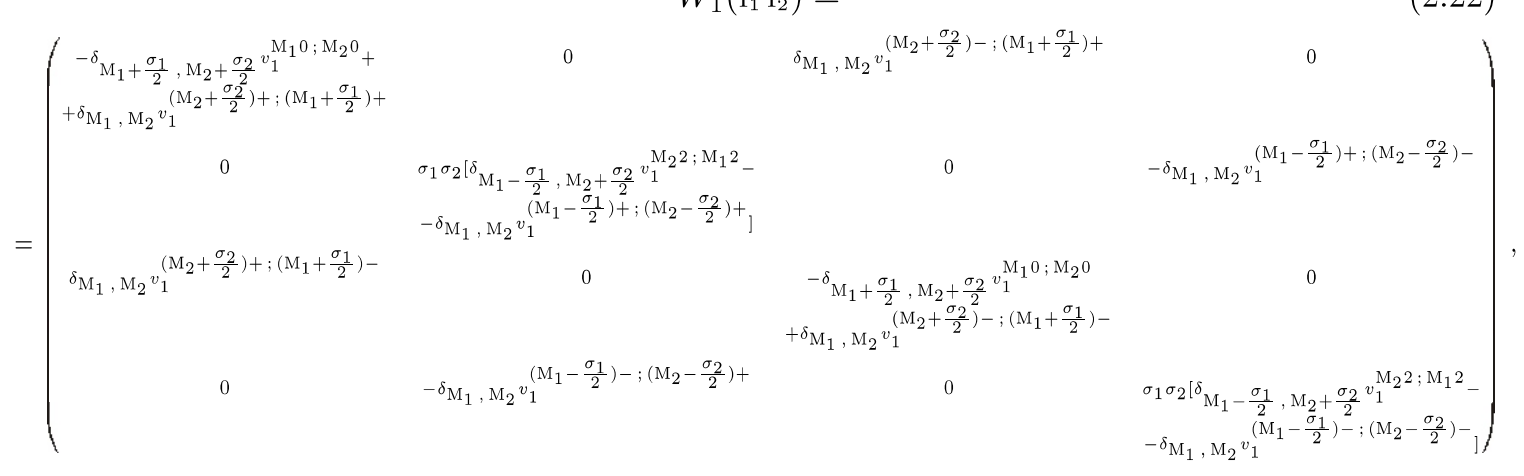




$$
\begin{aligned}
& \left(W_{1}^{02}\right)\left(\mathrm{I}_{1} \mathrm{I}_{2}\right)=
\end{aligned}
$$

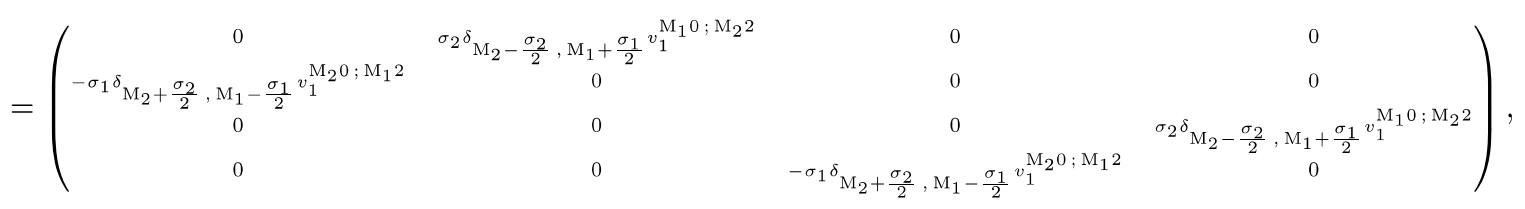

with symmetry relation

$$
\left(W^{\dagger}\right)^{02}\left(\mathrm{I}_{1} \mathrm{I}_{2}\right)=W^{20}\left(\mathrm{I}_{2} \mathrm{I}_{1}\right) .
$$

Now we are ready to write down an explicit form of equation for

$$
\mathcal{G}_{12}\left(\mathrm{I}_{1} \mathrm{I}_{2}\right) \equiv-\left\langle T \Psi_{1 \sigma_{1}}\left(\mathrm{I}_{1}\right) \Psi_{2 \sigma_{2}}^{\dagger}\left(\mathrm{I}_{2}\right)\right\rangle_{V} .
$$

\section{Equation for matrix electron Green's function}

Equation of motion for matrix GF (2.9) in terms of functional derivatives can be obtained in GFA similar to the Hubbard model [9]. It has a standard form [5]:

$$
\left[\left(L_{0 V}^{-1}\right)_{11^{\prime}}^{\sigma_{1} \sigma_{1}^{\prime}}\left(\mathrm{M}_{1} \mathrm{M}_{1}^{\prime}\right)-(\widehat{A} \Phi Y)_{11^{\prime}}^{\sigma_{1} \sigma_{1}^{\prime}}\left(\mathrm{M}_{1} \mathrm{M}_{1}^{\prime}\right)-(\widehat{A} Y)_{11^{\prime}}^{\sigma_{1} \sigma_{1}^{\prime}}\left(\mathrm{M}_{1} \mathrm{M}_{1}^{\prime}\right)\right] \mathcal{L}_{1^{\prime} 2}^{\sigma_{1}^{\prime} \sigma_{2}}\left(\mathrm{M}_{1}^{\prime} \mathrm{M}_{2}\right)=(\widehat{A} \Phi)_{12}^{\sigma_{1} \sigma_{2}}\left(\mathrm{M}_{1} \mathrm{M}_{2}\right)
$$

Here all quantities are $8 \times 8$-matrices. $L_{0 V}$ is $\mathrm{GF}$ of zeroth approximation in the fluctuating fields. We present it in the form

$$
\left(L_{0 V}^{-1}\right)_{12}^{\sigma_{1} \sigma_{2}}\left(\mathrm{M}_{1} \mathrm{M}_{2}\right)=\left(\begin{array}{cc}
G_{0}^{-1} \delta_{\sigma_{1} \sigma_{2}} \delta_{\mathrm{M}_{1}, \mathrm{M}_{2}}-W^{\sigma_{1} \sigma_{2}}\left(\mathrm{M}_{1} \mathrm{M}_{2}\right) & \left(W^{02}\right)^{\sigma_{1} \sigma_{2}}\left(\mathrm{M}_{1} \mathrm{M}_{2}\right) \\
-\left(W^{20}\right)^{\sigma_{1} \sigma_{2}}\left(\mathrm{M}_{1} \mathrm{M}_{2}\right) & \widetilde{G}_{0}^{-1} \delta_{\sigma_{1} \sigma_{2}} \delta_{\mathrm{M}_{1}, \mathrm{M}_{2}}+\widetilde{W}^{\sigma_{1} \sigma_{2}}\left(\mathrm{M}_{1} \mathrm{M}_{2}\right)
\end{array}\right) \delta_{12} .
$$

Matrix $Y$ determines the effective hopping of an electron on the lattice

$$
Y_{12}^{\sigma_{1} \sigma_{2}}\left(\mathrm{M}_{1} \mathrm{M}_{2}\right)=t_{12}\left(\begin{array}{cc}
\mathbb{T}^{\sigma_{1} \sigma_{2}}\left(\mathrm{M}_{1} \mathrm{M}_{2}\right) & 0 \\
0 & -\widetilde{\mathbb{T}}^{\sigma_{1} \sigma_{2}}\left(\mathrm{M}_{1} \mathrm{M}_{2}\right)
\end{array}\right)
$$

and matrix $\widehat{A}$ includes functional derivatives over the fields. It is convinient to write it as follows:

$$
\widehat{A}_{12}^{\sigma_{1} \sigma_{2}}\left(\mathrm{M}_{1} \mathrm{M}_{2}\right)=\delta_{12}\left(\begin{array}{cc}
\widehat{\mathcal{F}}_{1}^{\sigma_{1} \sigma_{2}}\left(\mathrm{M}_{1} \mathrm{M}_{2}\right) & \widehat{\mathcal{Q}}_{1}^{\sigma_{1} \sigma_{2}}\left(\mathrm{M}_{1} \mathrm{M}_{2}\right) \\
\widehat{\mathcal{Q}}_{1}^{\dagger \sigma_{1} \sigma_{2}}\left(\mathrm{M}_{1} \mathrm{M}_{2}\right) & \widehat{\mathcal{F}}_{1}^{\dagger \sigma_{1} \sigma_{2}}\left(\mathrm{M}_{1} \mathrm{M}_{2}\right)
\end{array}\right) \equiv \delta_{12} \widehat{A}_{1}^{\sigma_{1} \sigma_{2}}\left(\mathrm{M}_{1} \mathrm{M}_{2}\right)
$$

Quantities $\widehat{\mathcal{F}}_{1}, \widehat{\mathcal{Q}}_{1}, \widehat{\mathcal{F}}_{1}^{\dagger}$ and $\widehat{\mathcal{Q}}_{1}^{\dagger}$ are determined in equations $(2.17)-(2.20)$ in which $X$-operators should be replaced by functional derivatives according to a receipe:

$$
X_{1}^{p q} \rightarrow-\frac{\delta}{\delta v_{1}^{p q}} .
$$

At last we have to add definitions for GFs of zero approximation $G_{0}$ and $\widetilde{G}_{0}$

$$
G_{0}^{-1}=-\frac{\mathrm{d}}{\mathrm{d} \tau_{1}}-\mathcal{E}, \quad \widetilde{G}_{0}^{-1}=-\frac{\mathrm{d}}{\mathrm{d} \tau_{1}}+\mathcal{E},
$$

where diagonal matrix $\mathcal{E}$ is given by equation $(2.21)$.

Notice that equation (3.1) coincides in this form with equations for electron GFs for all basic models of strongly correlated systems [5]. Iterations in this equation generate a perturbation theory near the atomic limit. 
Having matrix elements for GF constructed on $X$-operators we are able to write down the electron GF determined on Fermi operators. To this end, keeping in mind formula (2.3) we must sum up over indexes $\mathrm{M}_{1}$ and $\mathrm{M}_{2}$ giving the states of atoms. It is reasonable to do this summation directly in equation (3.1). Then one can obtain the equation for "averaged" GF if we define it by relation:

$$
\overline{\mathcal{L}}_{12}^{\sigma_{1} \sigma_{2}}=\sum_{\mathrm{M}_{1} \mathrm{M}_{2}} \theta^{\sigma_{1}}\left(\mathrm{M}_{1}\right) \mathcal{L}_{12}^{\sigma_{1} \sigma_{2}}\left(\mathrm{M}_{1} \mathrm{M}_{2}\right) \theta^{\sigma_{2}}\left(\mathrm{M}_{2}\right)
$$

where

$$
\theta^{\sigma}(\mathrm{M})=\left(\begin{array}{cc}
\vartheta^{\sigma}(\mathrm{M}) & 0 \\
0 & \vartheta^{\sigma}(\mathrm{M})
\end{array}\right)
$$

with diagonal matrix $\vartheta^{\sigma}(\mathrm{M})$ (2.8). Multiplicative character of matrix (2.7), for the electron hopping on the lattice makes it possible to derive an equation for averaged GF $\overline{\mathcal{L}}$. We can write this equation as follows:

$$
\left[\left(\bar{L}_{0 V}^{-1}\right)_{11^{\prime}}^{\sigma_{1} \sigma_{1}^{\prime}}-\left(\widehat{\bar{A}}_{1}^{\sigma_{1} \sigma_{1}^{\prime}} \Phi\right) \mathcal{T}_{11^{\prime}}-\widehat{\bar{A}}_{1}^{\sigma_{1} \sigma_{1}^{\prime}} \mathcal{T}_{11^{\prime}}\right] \overline{\mathcal{L}}_{1^{\prime} 2}^{\sigma_{1}^{\prime} \sigma_{2}}=\delta_{12}\left(\widehat{\bar{A}}_{1}^{\sigma_{1} \sigma_{2}} \Phi\right)
$$

Here all overlined quantities are determined by the relations of type (3.7). For example operator $\widehat{\bar{A}}_{1}$ and effective hopping $\mathcal{T}_{12}$ are given by matrices:

$$
\begin{gathered}
\widehat{\widehat{A}}_{1}^{\sigma_{1} \sigma_{2}}=\left(\begin{array}{cc}
\hat{\mathcal{F}}_{1}^{\sigma_{1} \sigma_{2}} & \widehat{\overline{\mathcal{Q}}}_{1}^{\sigma_{1} \sigma_{2}} \\
\widehat{\overline{\mathcal{Q}}}_{1}^{\dagger \sigma_{1} \sigma_{2}} & \widehat{\widehat{\mathcal{F}}}_{1}^{\dagger \sigma_{1} \sigma_{2}}
\end{array}\right), \\
\mathcal{T}_{12}=t_{12}\left(\begin{array}{cc}
\mathrm{T} & 0 \\
0 & -\mathrm{T}
\end{array}\right),
\end{gathered}
$$

(matrix T was defined following the equation (2.8)). In equation (3.10) quantity $\widehat{\overline{\mathcal{F}}}$ is defined by relation:

$$
\widehat{\overline{\mathcal{F}}}_{1}^{\sigma_{1} \sigma_{2}}=\sum_{\mathrm{M}_{1} \mathrm{M}_{2}} \vartheta^{\sigma_{1}}\left(\mathrm{M}_{1}\right) \widehat{\mathcal{F}}_{1}^{\sigma_{1} \sigma_{2}}\left(\mathcal{M}_{1} \mathrm{M}_{2}\right) \vartheta^{\sigma_{2}}\left(\mathrm{M}_{2}\right)
$$

and so on.

An explicit form of matrix elements $\widehat{\overline{\mathcal{F}}}, \widehat{\widehat{\mathcal{Q}}}$ and their conjugated ones can be easily written if necessary. Notice only that for $4 \times 4$ matrix $\frac{\widehat{\mathcal{F}}}{\widehat{\mathcal{F}}}$

$$
\widehat{\widehat{\mathcal{F}}}^{12}=\widehat{\widehat{\mathcal{F}}}^{14}=\widehat{\widehat{\mathcal{F}}}^{21}=\widehat{\widehat{\mathcal{F}}}^{23}=\widehat{\widehat{\mathcal{F}}}^{32}=\widehat{\widehat{\mathcal{F}}}^{34}=\widehat{\overline{\mathcal{F}}}^{41}=\widehat{\widehat{\mathcal{F}}}^{43}=0,
$$

and in matrix $\widehat{\overline{\mathcal{Q}}}$ only elements

$$
\widehat{\overline{\mathcal{Q}}}^{12}, \widehat{\overline{\mathcal{Q}}}^{21}, \widehat{\overline{\mathcal{Q}}}^{34}, \widehat{\overline{\mathcal{Q}}}^{23}
$$

do not vanish (spin indexes $\sigma_{1}$ and $\sigma_{2}$ are dropped).

Zero order GF $L_{0 V}$ in the fluctuating fields is given by a relation

$$
\begin{gathered}
\left(\bar{L}_{0 V}^{-1}\right)_{12}^{\sigma_{1} \sigma_{2}}=\delta_{12}\left(\begin{array}{cc}
G_{0}^{-1} \delta_{\sigma_{1} \sigma_{2}}-\bar{W}^{\sigma_{1} \sigma_{2}} \mathbb{S}^{-1} & \left(\bar{W}^{02}\right)^{\sigma_{1} \sigma_{2} \mathbb{S}^{-1}} \\
-\left(\bar{W}^{20}\right)^{\sigma_{1} \sigma_{2} \mathbb{S}^{-1}} & \widetilde{G}_{0}^{-1} \delta_{\sigma_{1} \sigma_{2}}-\overline{\bar{W}}^{\sigma_{1} \sigma_{2}} \mathbb{S}^{-1}
\end{array}\right) \\
\mathbb{S}=\operatorname{diag}\{S+1, S+1, S, S\}
\end{gathered}
$$

\section{Solution of equation for the electron Green's function}

Equation (3.9) has a standard form of equation for basic models of strongly correlated systems. As usual we look for its solution in the multiplicative form:

$$
\overline{\mathcal{L}}=\bar{L} \bar{\Pi},
$$


where a propagator $L$ obeys the Dyson equation

$$
\bar{L}^{-1}=\bar{L}_{0 V}^{-1}-\bar{\Sigma}, \quad \bar{\Sigma}=\bar{\Sigma}^{\prime}+\bar{\Pi} \bar{Y} .
$$

Here in the expression for the self-energy part $\bar{\Sigma}$ we extracted a term $\bar{\Pi} \bar{Y}$, which can be cut over "the interaction line" $\bar{Y}$. From the basic equation (3.9) one obtains a coupled equation for the terminal part $\bar{\Pi}$ and uncutable self-energy part $\bar{\Sigma}^{\prime}$ of the GF $\overline{\mathcal{L}}$. We write them down in a form, where

$$
\begin{aligned}
\bar{\Pi}(12) & =(\widehat{\bar{A}} \Phi)(12)+(\bar{Y} \bar{L})\left(4^{\prime} 3^{\prime}\right) \widehat{\bar{A}}\left(14^{\prime}\right) \bar{\Pi}\left(3^{\prime} 2\right) \\
\bar{\Sigma}^{\prime}(12) & =-(\bar{Y} \bar{L})\left(4^{\prime} 3^{\prime}\right) \widehat{\bar{A}}\left(14^{\prime}\right)\left[\bar{L}_{0 V}^{-1}\left(3^{\prime} 2\right)-\bar{\Sigma}^{\prime}\left(3^{\prime} 2\right)\right] .
\end{aligned}
$$

If we are interested only in the normal GF (first matrix element in (2.9)) which we denote by $\overline{\mathcal{G}}$, corresponding to $\overline{\mathcal{G}}$ propagator part of GF is $\bar{G}$, and its zero order approximation is $\bar{G}_{0 V}$, terminal part is $\Lambda$ and self-energy part is $\bar{\Sigma}$, then from general equation (4.1)-(4.4) it follows

$$
\begin{aligned}
\overline{\mathcal{G}}_{12}^{\sigma_{1} \sigma_{2}} & =\bar{G}_{11^{\prime}}^{\sigma_{1} \sigma_{1}^{\prime}} \bar{\Lambda}_{1^{\prime} 2}^{\sigma_{1}^{\prime} \sigma_{2}}, \\
\left(\bar{G}^{-1}\right)_{12}^{\sigma_{1} \sigma_{2}} & =\left(\bar{G}_{0 V}^{-1}\right)_{12}^{\sigma_{1} \sigma_{2}}-\bar{\Sigma}_{12}^{\sigma_{1} \sigma_{2}}, \quad \bar{\Sigma}_{12}^{\sigma_{1} \sigma_{2}}=\bar{\Sigma}_{12}^{\prime \sigma_{1} \sigma_{2}}-(\bar{\Lambda} \mathrm{T})_{12}^{\sigma_{1} \sigma_{2}} .
\end{aligned}
$$

Here all quantities $\overline{\mathcal{G}}, \bar{G}, \bar{G}_{0 V}, \bar{\Lambda}, \bar{\Sigma}, \bar{\Sigma}^{\prime}$ and T are $4 \times 4$-matrices.

First iteration in equation (4.3) leads to an expression for self-energy in the first and second order, over hopping respectively. Let us calculate the self-energy part

$$
\bar{\Sigma}_{12}^{\sigma \sigma}=(\bar{\Lambda} \mathrm{T})_{12}^{\sigma \sigma} .
$$

Taking the iteration expression of the terminal part $\bar{\Pi}$ one can write an expression for $\bar{\Sigma}$ :

$$
\bar{\Sigma}_{12}^{\sigma \sigma}=\left(\bar{\Lambda}^{(0)} \mathrm{T}\right)^{\sigma \sigma} t_{12}+\left[\hat{\overline{\mathcal{F}}}_{1}^{\sigma \sigma^{\prime}} \mathcal{T}(t \bar{G})_{12^{\prime}}^{\sigma^{\prime} \sigma^{\prime}} \hat{\overline{\mathcal{F}}}_{2^{\prime}}^{\sigma^{\prime} \sigma} t_{2^{\prime} 2}-\hat{\overline{\mathcal{Q}}}_{1}^{\sigma \sigma^{\prime}} \mathrm{T}(t \overline{\widetilde{G}})_{12^{\prime}}^{\sigma^{\prime} \sigma^{\prime}} \hat{\mathcal{Q}}_{2^{\prime}}^{\dagger \sigma^{\prime} \sigma} t_{2^{\prime} 2}\right] \Phi .
$$

Each matrix here is a $4 \times 4$-matrix. After substituting expressions for $\bar{F}$ and $\bar{Q}$ one can write a result for $\bar{\Sigma}_{12}^{\sigma \sigma}$ in a form:

$$
\bar{\Sigma}=\left(\begin{array}{cccc}
\lambda_{1} & \lambda_{1} & \lambda_{1} & \lambda_{1} \\
\lambda_{2} & \lambda_{2} & \lambda_{2} & \lambda_{2} \\
\lambda_{3} & \lambda_{3} & \lambda_{3} & \lambda_{3} \\
\lambda_{4} & \lambda_{4} & \lambda_{4} & \lambda_{4}
\end{array}\right)
$$

where

$$
\begin{aligned}
& \left(\lambda_{1}\right)_{12}^{\sigma \sigma}=\left\langle\overline{\mathcal{F}}_{1}^{11}+\overline{\mathcal{F}}_{1}^{13}\right\rangle^{\sigma \sigma} t_{12}+\left\langle T\left(\overline{\mathcal{F}}_{1}^{11}+\overline{\mathcal{F}}_{1}^{13}\right)^{\sigma \sigma^{\prime}} A_{12^{\prime}}^{\sigma^{\prime} \sigma}\right\rangle t_{2^{\prime} 2}-\left\langle T\left(\overline{\mathcal{Q}}_{1}^{12}\right)^{\sigma \sigma^{\prime}} B_{12^{\prime}}^{\sigma^{\prime} \sigma}\right\rangle t_{2^{\prime} 2}, \\
& \left(\lambda_{2}\right)_{12}^{\sigma \sigma}=\left\langle\overline{\mathcal{F}}_{1}^{22}+\overline{\mathcal{F}}_{1}^{24}\right\rangle^{\sigma \sigma} t_{12}+\left\langle T\left(\overline{\mathcal{F}}_{1}^{22}+\overline{\mathcal{F}}_{1}^{24}\right)^{\sigma \sigma^{\prime}} A_{12^{\prime}}^{\sigma^{\prime} \sigma}\right\rangle t_{2^{\prime} 2}-\left\langle T\left(\overline{\mathcal{Q}}_{1}^{21}\right)^{\sigma \sigma^{\prime}} B_{12^{\prime}}^{\sigma^{\prime} \sigma}\right\rangle t_{2^{\prime} 2}, \\
& \left(\lambda_{3}\right)_{12}^{\sigma \sigma}=\left\langle\overline{\mathcal{F}}_{1}^{33}+\overline{\mathcal{F}}_{1}^{31}\right\rangle^{\sigma \sigma} t_{12}+\left\langle T\left(\overline{\mathcal{F}}_{1}^{33}+\overline{\mathcal{F}}_{1}^{31}\right)^{\sigma \sigma^{\prime}} A_{12^{\prime}}^{\sigma^{\prime} \sigma}\right\rangle t_{2^{\prime} 2}-\left\langle T\left(\overline{\mathcal{Q}}_{1}^{34}\right)^{\sigma \sigma^{\prime}} B_{12^{\prime}}^{\sigma^{\prime} \sigma}\right\rangle t_{2^{\prime} 2}, \\
& \left(\lambda_{4}\right)_{12}^{\sigma \sigma}=\left\langle\overline{\mathcal{F}}_{1}^{44}+\overline{\mathcal{F}}_{1}^{42}\right\rangle^{\sigma \sigma} t_{12}+\left\langle T\left(\overline{\mathcal{F}}_{1}^{44}+\overline{\mathcal{F}}_{1}^{42}\right)^{\sigma \sigma^{\prime}} A_{12^{\prime}}^{\sigma^{\prime} \sigma}\right\rangle t_{2^{\prime} 2}-\left\langle T\left(\overline{\mathcal{Q}}_{1}^{43}\right)^{\sigma \sigma^{\prime}} B_{12^{\prime}}^{\sigma^{\prime} \sigma}\right\rangle t_{2^{\prime} 2} .
\end{aligned}
$$

Here we introduce a notation:

$$
\begin{aligned}
A_{12^{\prime}}^{\sigma^{\prime} \sigma}= & \left(t G^{(1)}\right)_{12^{\prime}}^{\sigma^{\prime} \sigma^{\prime}}\left(\overline{\mathcal{F}}_{2^{\prime}}^{11}+\overline{\mathcal{F}}_{2^{\prime}}^{13}\right)^{\sigma^{\prime} \sigma}+\left(t G^{(2)}\right)_{12^{\prime}}^{\sigma^{\prime} \sigma^{\prime}}\left(\overline{\mathcal{F}}_{2^{\prime}}^{22}+\overline{\mathcal{F}}_{2^{\prime}}^{24}\right)^{\sigma^{\prime} \sigma} \\
& +\left(t G^{(3)}\right)_{12^{\prime}}^{\sigma^{\prime} \sigma^{\prime}}\left(\overline{\mathcal{F}}_{2^{\prime}}^{33}+\overline{\mathcal{F}}_{2^{\prime}}^{31}\right)^{\sigma^{\prime} \sigma}+\left(t G^{(4)}\right)_{12^{\prime}}^{\sigma^{\prime} \sigma^{\prime}}\left(\overline{\mathcal{F}}_{2^{\prime}}^{44}+\overline{\mathcal{F}}_{2^{\prime}}^{42}\right)^{\sigma^{\prime} \sigma}, \\
B_{12^{\prime}}^{\sigma^{\prime} \sigma}= & \left(t \widetilde{G}^{(1)}\right)_{12^{\prime}}^{\sigma^{\prime} \sigma^{\prime}}\left(\overline{\mathcal{Q}}_{2^{\prime}}^{\dagger 12}\right)^{\sigma^{\prime} \sigma}+\left(t \widetilde{G}^{(2)}\right)_{12^{\prime}}^{\sigma^{\prime} \sigma^{\prime}}\left(\overline{\mathcal{Q}}_{2^{\prime}}^{\dagger 21}\right)^{\sigma^{\prime} \sigma} \\
& +\left(t \widetilde{G}^{(3)}\right)_{12^{\prime}}^{\sigma^{\prime} \sigma^{\prime}}\left(\overline{\mathcal{Q}}_{2^{\prime}}^{\dagger 34}\right)^{\sigma^{\prime} \sigma}+\left(t \widetilde{G}^{(4)}\right)_{12^{\prime}}^{\sigma^{\prime} \sigma^{\prime}}\left(\overline{\mathcal{Q}}_{2^{\prime}}^{\dagger 43}\right)^{\sigma^{\prime} \sigma} .
\end{aligned}
$$


We see that the first order terms in $\lambda_{i}(i=1,2,3,4)$ are expressed by averages of $X$-operators and the second order terms by $T$-products of two $X$-operators, being bose-type GFs.

In equations (4.9) and (4.10) some combinations of electron GFs were introduced, namely

$$
\left(G^{(j)}\right)_{12}^{\sigma \sigma}=\sum_{i}\left(G^{(i j)}\right)_{12}^{\sigma \sigma}
$$

We shall look for a solution of Dyson equation for propagator $\bar{G}^{\sigma}(k)$, when the self-energy $\bar{\Sigma}$ is determined by equation 4.7) and equation (4.8). Due to a specific matrix (4.8), the solution can be written as

$$
\bar{G}^{\sigma}(k)=\left[1+\frac{1}{d_{s}(k)} G_{0}^{\sigma}(k) \bar{\Lambda}^{\sigma} \mathrm{T} \varepsilon_{\boldsymbol{k}}\right] G_{0}^{\sigma}(k),
$$

where

$$
d_{s}(k)=1-\left(\frac{\lambda_{1}(k)}{z_{n}-E_{+}}+\frac{\lambda_{2}(k)}{z_{n}+E_{+}}+\frac{\lambda_{3}(k)}{z_{n}-E_{-}}+\frac{\lambda_{4}(k)}{z_{n}+E_{-}}\right),
$$

$z_{n}=\mathrm{i} \omega_{n}+\mu$. Explicit expressions for matrices $\bar{G}$ and $\overline{\mathcal{G}}$ are complicated and we do not write them down.

One can see that in expressions for $\lambda_{i}$ determining the electron GF, some linear combinations of $X$-operators may appear which determine the components of the total spin on a site $\boldsymbol{S}_{i}^{\text {tot }}=\boldsymbol{S}_{i}+\frac{1}{2} \boldsymbol{\sigma}$ $[1,2]$ :

$$
\begin{aligned}
& S_{\mathrm{tot}}^{z}=\sum_{\mathrm{M}=-S}^{S} \mathrm{M}\left(X^{\mathrm{M} 0 ; \mathrm{M} 0}+X^{\mathrm{M} 2 ; \mathrm{M} 2}\right)+\sum_{\alpha} \sum_{\mathrm{M}^{\alpha}=-\left(S+\frac{\alpha}{2}\right)}^{S+\frac{\alpha}{2}} \mathrm{M}^{\alpha} X^{\mathrm{M}^{\alpha} \alpha ; \mathrm{M}^{\alpha} \alpha} \\
& S_{\mathrm{tot}}^{\sigma}=\sum_{\mathrm{M}=-S}^{S} \nu_{S}^{\sigma}(\mathrm{M})\left(X^{(\mathrm{M}+\sigma) 0 ; \mathrm{M} 0}+X^{(\mathrm{M}+\sigma) 2 ; \mathrm{M} 2}\right)+\sum_{\alpha} \sum_{\mathrm{M}^{\alpha}=-\left(S+\frac{\alpha}{2}\right)}^{S} \nu_{S+\frac{\alpha}{2}}^{\sigma}\left(\mathrm{M}^{\alpha}\right) X^{\left(\mathrm{M}^{\alpha}+\sigma\right) \alpha ; \mathrm{M}^{\alpha} \alpha}
\end{aligned}
$$

where

$$
\nu_{s}^{\sigma}(\mathrm{M})=\frac{1}{\sqrt{2}} \sqrt{(S-\sigma \mathrm{M})(S+\sigma \mathrm{M}+1)},
$$

as well as the components for the pseudospin [5]

$$
\begin{aligned}
& \rho^{z}=\sum_{\mathrm{M}=-S}^{S}\left(X^{\mathrm{M} 2 ; \mathrm{M} 2}-X^{\mathrm{M} 0 ; \mathrm{M} 0}\right), \\
& \rho^{+}=\sum_{\mathrm{M}=-S}^{S} X^{\mathrm{M} 2 ; \mathrm{M} 0} ; \rho^{-}=\left(\rho^{+}\right)^{\dagger} .
\end{aligned}
$$

Along with these combinations in expressions for $\lambda_{i}$ there are terms containing operators of the type

$$
X^{\mathrm{M}^{\alpha} \alpha ; \mathrm{M}^{\bar{\alpha}} \bar{\alpha}},
$$

which describe the transfers on a site with change of the total spin $S+\frac{\alpha}{2}$ on $S-\frac{\alpha}{2}$. At large values of sd-exchange integral such transfers give a small contribution to statistical averages. We shall neglect such terms. Therefore, all off diagonal matrix elements $\overline{\mathcal{F}}^{i j}$ can be omitted. In the rest of the expressions for $\lambda_{i}$, the following combinations of matrix elements appear:

$$
\begin{aligned}
& \left(\overline{\mathcal{F}}_{1}^{11}+\overline{\mathcal{F}}_{1}^{44}\right)^{\sigma_{1} \sigma_{2}}=\delta_{12} \frac{1}{2}\left(1+\frac{2 \sigma_{1}}{2 S+1} S_{1 \text { tot }}^{z}-\frac{1}{2 S+1} \rho_{1}^{z}\right)+\delta_{\bar{\sigma}_{1} \sigma_{2}} \frac{\sqrt{2}}{2 S+1} S_{1 \text { tot }}^{\bar{\sigma}_{1}} \\
& \left(\overline{\mathcal{F}}_{1}^{22}+\overline{\mathcal{F}}_{1}^{33}\right)^{\sigma_{1} \sigma_{2}}=\delta_{12} \frac{1}{2}\left(1-\frac{2 \sigma_{1}}{2 S+1} S_{1 \text { tot }}^{z}-\frac{1}{2 S+1} \rho_{1}^{z}\right)-\delta_{\bar{\sigma}_{1} \sigma_{2}} \frac{\sqrt{2}}{2 S+1} S_{1 \text { tot }}^{\bar{\sigma}_{1}},
\end{aligned}
$$




$$
\begin{gathered}
\left(\overline{\mathcal{Q}}_{1}^{12}+\overline{\mathcal{Q}}_{1}^{43}\right)^{\sigma_{1} \sigma_{2}}=-\left(\overline{\mathcal{Q}}_{1}^{21}+\overline{\mathcal{Q}}_{1}^{34}\right)^{\sigma_{1} \sigma_{2}}=-\delta_{\bar{\sigma}_{1} \sigma_{2}} \frac{1}{2 S+1} \rho_{1}^{-}, \\
\left(\overline{\mathcal{Q}}_{1}^{\dagger 12}+\overline{\mathcal{Q}}_{1}^{\dagger 3}\right)^{\sigma_{1} \sigma_{2}}=-\left(\overline{\mathcal{Q}}_{1}^{\dagger 21}+\overline{\mathcal{Q}}_{1}^{\dagger 34}\right)^{\sigma_{1} \sigma_{2}}=\delta_{\bar{\sigma}_{1} \sigma_{2}} \frac{\sigma_{1}}{2 S+1} \rho_{1}^{+} .
\end{gathered}
$$

We see that in expressions for $\lambda_{i}$, in fact, there appear components of total spin and pseudospin. However, some combinations of $X$-operators of the type (4.19) still remain. Their role, however, decreases with parameter $J$ increasing. Further proceedings with an analytical study can be done in a limit of large atomic spin $S$.

\section{Limit $S=\infty$}

In this limit both eigenvalues of the single site sd-exchange Hamiltonian coincide, so that $\left|E_{-}\right|=\left|E_{+}\right|=\frac{S J}{2}$ and a condition $d_{s}(k)=0$ for poles of the electron GF reduce to an equation of second order but not fourth order as in general case. In this situation, matrix (4.13) and electron GF constructed on Fermi operators can be easily written explicitly. Using the identity

$$
g^{\sigma}(k)=-\left\langle T c_{1 \sigma} c_{2 \sigma}^{\dagger}\right\rangle=\sum_{i j}\left[\overline{\mathcal{G}}^{\sigma}(k)\right]^{i j},
$$

we can write the result of the calculation in the following form

$$
g^{\sigma}(k)=\frac{1}{F^{\sigma}(k)-\varepsilon_{\boldsymbol{k}}},
$$

where

$$
F^{\sigma}(k)=\frac{z_{n}^{2}-(I / 2)^{2}}{z_{n}\left(\lambda_{1}^{\sigma}+\lambda_{2}^{\sigma}+\lambda_{3}^{\sigma}+\lambda_{4}^{\sigma}\right)-I / 2\left(\lambda_{1}^{\sigma}+\lambda_{4}^{\sigma}-\lambda_{2}^{\sigma}-\lambda_{3}^{\sigma}\right)},
$$

and all quantities $\lambda_{i}^{\sigma} \equiv \lambda_{i}^{\sigma}(k)$ depend on spin and 4-momentum. We introduced notation $I=J S$, which should be finite in the limit $S=\infty$.

Linear combination of $\lambda_{i}$ in formula (5.3) in the site representation is equal to:

$$
\begin{aligned}
\left(\lambda_{1}^{\sigma}+\lambda_{2}^{\sigma}+\lambda_{3}^{\sigma}+\lambda_{4}^{\sigma}\right)_{12}^{\sigma \sigma}= & \delta_{12}, \\
\left(\lambda_{1}^{\sigma}+\lambda_{4}^{\sigma}-\lambda_{3}^{\sigma}-\lambda_{4}^{\sigma}\right)_{12}^{\sigma \sigma}= & \sigma\left\langle S_{1}^{z}\right\rangle t_{12}+\frac{1}{2}\left[t \bar{G}^{(1)}-t \bar{G}^{(2)}\right]_{12^{\prime}}^{\sigma \sigma}\left\langle T S_{2^{\prime}}^{z} S_{1}^{z}\right\rangle t_{2^{\prime} 2} \\
& +\left[t \bar{G}^{(1)}-t \bar{G}^{(2)}\right]_{12^{\prime}}^{\bar{\sigma} \bar{\sigma}}\left\langle T S_{2^{\prime}}^{\sigma} S_{1}^{\bar{\sigma}}\right\rangle t_{2^{\prime} 2},
\end{aligned}
$$

where we introduced normalized spin operators $S_{1}^{\alpha}=\frac{1}{S} S_{1 \text { tot }}^{\alpha}$.

In momentum representation, the expressions (5.4) and (5.5) are given

$$
\begin{aligned}
\lambda_{1}(k)+\lambda_{2}(k)+\lambda_{3}(k)+\lambda_{4}(k) & =1, \\
\lambda_{1}(k)+\lambda_{4}(k)-\lambda_{2}(k)-\lambda_{3}(k) & =\varepsilon_{\boldsymbol{k}} \sum_{q} \varepsilon_{\boldsymbol{k}+\boldsymbol{q}} G(k+q) \mathcal{D}(q) \equiv \xi(k) \varepsilon_{\boldsymbol{k}},
\end{aligned}
$$

where $\mathcal{D}(q)$ is a Fourier-component of GF for spin fluctuations

$$
\mathcal{D}_{12}=-\left\langle T \boldsymbol{S}_{1} \boldsymbol{S}_{2}\right\rangle .
$$

In paramagnetic phase, electron GF $g(k)$ and a function $G(k)$, involved in (5.7) can be written in a form

$$
\begin{aligned}
g(k) & =\frac{z_{n}-\xi(k) I / 2}{z_{n}^{2}-(I / 2)^{2}-\left[z_{n}-\xi(k) I / 2\right] \varepsilon_{\boldsymbol{k}}} . \\
G(k) & =\frac{I}{z_{n}^{2}-(I / 2)^{2}-\left[z_{n}-\xi(k) I / 2\right] \varepsilon_{\boldsymbol{k}}},
\end{aligned}
$$


where $k=\left\{\boldsymbol{k}, \mathrm{i} \omega_{n}\right\} \cdot G(k)$ denotes a Fourier transform of the quantity $\left[t \bar{G}^{(1)}-t \bar{G}^{(2)}\right]$ involved in expression (5.5).

Using the spectral representation for Fermi-like GFs $g(k)$ and $G(k)$ and Bose-like GF $\mathcal{D}(k)$ we present the expression (5.7) for $\xi(k)$ in a form

$$
\xi(k)=-\frac{1}{N} \sum_{\boldsymbol{k}^{\prime}} \varepsilon_{\boldsymbol{k}^{\prime}} \int \frac{\omega^{\prime}}{\pi} \int \frac{\Omega^{\prime}}{\pi} \frac{\operatorname{Im} G\left(\boldsymbol{k}^{\prime}, \omega^{\prime}\right) \operatorname{Im} \mathcal{D}\left(\boldsymbol{k}^{\prime}-\boldsymbol{k}, \Omega^{\prime}\right)}{\mathrm{i} \omega_{n}-\omega^{\prime}+\Omega^{\prime}}\left(f\left[\omega^{\prime}\right]+n\left[\Omega^{\prime}\right]\right),
$$

where $f\left[\omega^{\prime}\right]$ and $n\left[\Omega^{\prime}\right]$ are functions of Fermi and Bose, respectively. After analytical continuation $\mathrm{i} \omega_{n} \rightarrow \omega+\mathrm{i} \delta$ we obtain an equation for $\xi_{\boldsymbol{k}}(\omega)$, which is an integral one over frequency and momentum. It should be solved together with the equation for chemical potential

$$
n=2 \frac{1}{N} \sum_{\boldsymbol{k}^{\prime}} \varepsilon_{\boldsymbol{k}^{\prime}} \int \omega^{\prime}\left[-\frac{1}{\pi} \operatorname{Im} g\left(\boldsymbol{k}^{\prime}, \omega^{\prime}\right)\right] f\left[\omega^{\prime}\right]
$$

Now we do an estimation of quantity $\xi(k)$ under the following approximation: consider limit of static fluctuations

$$
\operatorname{ImD}(\boldsymbol{q}, \Omega) \approx \pi a_{\boldsymbol{q}} \delta(\Omega)
$$

and neglect $\boldsymbol{q}$-dependence of the spectral density $a_{\boldsymbol{q}} \approx a$. Then for temperature $T=0$ from $(5.11)$ it follows

$$
\xi(\omega)=-\frac{a I}{W} \int_{-\frac{W}{2}}^{\bar{\varepsilon}} \mathrm{d} \varepsilon \frac{\varepsilon}{\varepsilon^{2}+I^{2}} \frac{1}{\omega+E_{1}(\bar{\varepsilon})-E_{1}(\varepsilon)},
$$

where we use a rectangular form of the bare band and mean field approximation for electron GF $g(k)$, equation (5.9) with $\xi(k)=0$.

$$
g(\boldsymbol{q}, \omega)=\frac{1}{2}\left(1-\frac{\varepsilon_{k}}{Q_{\boldsymbol{k}}}\right) \frac{1}{\omega+\mu-E_{1}(\boldsymbol{k})}+\frac{1}{2}\left(1+\frac{\varepsilon_{k}}{Q_{\boldsymbol{k}}}\right) \frac{1}{\omega+\mu-E_{2}(\boldsymbol{k})},
$$

with $Q_{\boldsymbol{k}}=E_{2}(\boldsymbol{k})-E_{1}(\boldsymbol{k})$, and

$$
E_{1,2}(\boldsymbol{k})=\frac{1}{2}\left(\varepsilon_{k} \mp \sqrt{\varepsilon_{k}^{2}+I^{2}}\right)
$$

determine two branches of quasiparticle spectrum.

When electron density $n \leqslant 1$ chemical potential $\mu$ lies in the lower band, and $\bar{\varepsilon}$ means energy $\varepsilon_{\boldsymbol{q}}$ corresponding to fixed $\mu ; \bar{\varepsilon}$ obeys equaion $E_{1}(\bar{\varepsilon})=\mu$. One can see that $\xi(\omega)$ has logarithmic singularity at $\omega=0$ for account of electrons near Fermi-level.

In conclusion, we notice that, contrary to paper [4], here we developed an approximation for the sd-model with strong sd-exchange coupling beyond the mean-field theory to take into account fluctuations in the system. Electron GF (5.9) contains contribution of magnetic fluctuations via $\xi(k)$-quantity. Further analysis of the problem reduces now to the calculation $\xi(k)$ as a function of momentum and frequency.

Preliminary analysis of equations (5.9)-(5.12) shows that at half filling a gap between two branches of quasiparticle spectrum vanishes at large enough exchange parameter $I$, and an insulatormetal phase transition occurs. A detailed numerical analysis of equations (5.9)-(5.12) at half filling and beyond it will be done elsewhere.

This work was supported by Russian Foundation for Support of Scientific School, grant NS4640.2006.2.

\section{References}

1. Erukhimov M.S., Ovchinnikov S.G., Phys. Stat. Sol. (b), 1984, 123, 105.

2. Erukhimov M.S., Ovchinnikov S.G., Physica C, 1989, 157, 209. 
3. Nagaev E.L. Physics of Magnetic Semiconductors. Nauka, Moscow, 1979.

4. Izyumov Yu.A., Chaschin N.I., Alexeev D.S., Condensed Matter Physics, 2005, 123, No. 4, 801.

5. Izyumov Yu.A., Chaschin N.I., Alexeev D.S. Theory of Strongly Correlated Systems. The generating Functional Approach, Regular and Chaotic Dynamics. Moscow-Izhevsk, 2006.

6. Anokhin A.O., Irkhin V.Yu., Phys. Stat. Sol. (b), 1991, 165, 129.

7. Anokhin A.O., Irkhin V.Yu., Katsnelson M.I., J. Phys.: Condens. Matter, 1991, 3, 1475.

8. Abrikosov A.A., Gorkov L.P., Dzyaloshinskii I.E. Methods of Quantum Field Theory in Statistical Physics. Dover, New York, 1975.

9. Izyumov Yu.A., Chaschin N.I., Alexeev D.S., Mancini F., Eur. Phys. J. B, 2005, 45, 69.

\title{
Sd-модель з сильною обмінною взаємодією та фазовим переходом метал-діелектрик
}

\author{
Ю.О.Ізюмов, Н.І.Чащін, Д.С.Алексеєв \\ Інститут фізики металів, вул. С. Ковалевської, 18, Єкатеринбург, Росія \\ Отримано 18 травня 2006 р.б в остаточному вигляді - 14 червня 2006 р.
}

Sd-обмінна модель (модель Кондо гратки) сформульована для сильної sd-взаємодії в рамках техніки $X$-операторів та підходу твірного функціоналу. $X$-оператори побудовані на базисі точних власних функцій одновузлового sd-обмінного гамільтоніану. Таке представлення дозволяє розвинути теорію збурень поблизу атомного рівня. Представлення локаторного типу було отримане для електронної функції Гріна. Власна енергія електронів включає взаємодію електронів та спінових флуктуацій. Отримано інтегральне рівняння для власної енергії в границі безмежних локалізованих спінів. Розв'язок цього рівняння в статичному наближенні для спінових флуктуацій веде до структури електронної функції Гріна, що виявляє фазовий перехід метал-діелектрик. Цей перехід $є$ схожим до переходу в моделі Хаббарда при половинному заповненні.

Ключові слова: модель Кондо гратки, сильноскорельовані електронні системи, фазові переходи метал-діелектрик

PACS: $71.10 .-w, 71.10 . F d, 71.27 .+a$ 
